der Anschluß der Institute in der französischen Zone im Jahre 1949 erfolgt war, trat die Forschungsstelle für Physik der Stratosphäre auch in den Verband der Max-Planck-Gesellschaft ein und wird nunmehr von dieser durch die Ländergemeinschaft finanziert.

Regener selbst wurde durch einstimmigen Beschluß des Senats zum Vizepräsidenten der MaxPlanck-Gesellschaft gewählt.

Dieses Amt gab und gibt ihm Gelegenheit, auch in den grundsätzlichen Fragen der Wissenschaftspflege und Forschung sowie in der Vertretung der Institute in der französischen Zone dem Präsidenten der Gesellschaft weitgehende und wertvolle Unterstützung zu leisten.

So wünschen die Gesellschaft und der Präsident ihrem verdienten Mitarbeiter und tatkräftigen Vizepräsidenten noch weiterhin viele Jahre recht erfolgreicher Arbeit.

Ich persönlich freue mich besonders, mit meinem lieben Freunde aus lange verklungenen Beriner Jahren durch unsere gemeinsame Arbeit in der Max-Planck-Gesellschaft rein menschlich und dienstlich nahe verbunden zu bleiben.

Otto Hahn

\title{
Über die erkenntnistheoretische Problemlage und den Gebrauch einer dreiwertigen Logik in der Quantenmechanik
}

\author{
Von Hans Reichenbach \\ University of California, Los Angeles \\ (Z. Naturforschg. 6 a, 569—575 [1951]; eingegangen am 29. Mai 1951) \\ Erich Regener zum 70. Geburtstage gewidmet
}

$W_{\text {ph }}$ enn die Physik heute mehr als je im Zentrum philosophischer Diskussionen steht, so hat dies seinen Grund in der Tatsache, daß die physikalische Forschung zu einer Revision gewisser sehr allgemeiner Prinzipien des Naturerkennens geführt hat. Diese philosophischen Resultate sind zunächst in Einsteins Relativitätstheorie deutlich geworden, welche uns gelehrt hat, den raum-zeitlichen Rahmen der physikalischen Welt in neuem Lichte zu sehen; es hat sich herausgestellt, daß die allzu einfachen Vorstellungen von Raum und Zeit, die sich im Zusammenhang der physikalischen Umwelt des täglichen Lebens entwikkelt haben, mit den Resultaten exakter Messungen auf optischem und elektromagnetischem Gebiet nicht mehr vereinbar waren. Aber die Umstellung grundlegender Begriffe, die da von uns verlangt wurde, erscheint heutzutage bereits geringfügig im Vergleich zu den Veränderungen, welche die Quantenmechanik für das physikalische Weltbild mit sich gebracht hat. Denn die Physik der Quanten verlangt von uns, den Gedanken der allgemeinen Kausalität aufzugeben, der für die klassische Physik, einschließlich der Relativitätstheorie, stets als das leitende Prinzip angesehen wurde, ohne welches Naturerkenntnis unmöglich erschien.

Aber auch hier wieder hat sich die Entwicklung in Stufen vollzogen. Die Kausalität aufzugeben, hieß zunächst nur, an Stelle strenger physikalischer Gesetze Wahrscheinlichkeitsgesetze einzuführen. Solche Perspektiven hatten sich ja schon für die Boltzmannsche Aufklärung des zweiten Hauptsatzes der Wärmetheorie ergeben. Was damals nur als eine Möglichkeit erschien, ist in $\mathrm{He}$ i s e nbergs Unbestimmtheitsrelation zur Gewißheit geworden: die Voraussage quantenmechanischer Geschehnisse ist an eine Genauigkeitsgrenze gebunden, die mit Plancks Wirkungsquantum verknüpft ist und uns zwingt, die Quantenerscheinungen als lediglich statistisch bestimmt aufzufassen. Diese Genauigkeitsgrenze hat nichts mit der Unvollkommenheit des menschlichen Beobachters zu tun; sie hat ihren Grund in der Struktur der physikalischen Welt und läßt sich als eine Beziehung zwischen physikalischen Geschehnissen formulieren, ohne daß dabei eine Bezugnahme auf einen Beobachter erforderlich ist. Der Lapla ce sche Übermensch, der jedes Elementarteilchen genau beobachten kann und 
dessen mathematischen Fähigkeiten keine Grenze gezogen ist, wäre da nicht besser daran als wir: auch er könnte nur $\mathrm{S}$ chrödingers $\psi$-Funktionen für die Naturbeschreibung benutzen und wäre deshalb an das Prinzip der umgekehrten Verknüpfung der Wahrscheinlichkeitsverteilungen kanonisch konjugierter Parameter gebunden, das in B or $\mathrm{ns}$ statistischer Deutung der $\psi$-Funktion und $\mathrm{He}$ is e $\mathrm{n}$ bergs Unbestimmtheitsrelation seinen Ausdruck gefunden hat.

Mit dieser Erweiterung der Kausalität könnte man sich sozusagen noch leichten Herzens abfinden. Wenn die Natur auch im Kleinen unbestimmt ist, so ist sie doch im Großen bestimmt, weil die statistische Regelmäßigkeit großer Zahlen eine hinreichend exakte Gesetzlichkeit für makroskopische Objekte garantiert. $\mathrm{Ob}$ die kleinsten Teile die Bewegung der Planeten oder vielmehr die Vorgänge eines Würfelspiels imitieren, erscheint nicht so wesentlich, solange im Großen dasselbe herauskommt. Jedenfalls bringt eine solche Veränderung für unsere Vorstellung von der Welt keine Schwierigkeiten mit sich. Das Gesetz der Kausalität ist ein Resultat der Erfahrung, und wir müssen bereit sein, es durch eine allgemeinere Form von Gesetzlichkeit zu ersetzen, wenn physikalische Beobachtungen es verlangen.

Es hat sich aber herausgestellt, daß diese Erweiterung der Kausalität nicht hinreichend ist, wenn man die quantenmechanischen Erscheinungen verstehen will. Es ist nicht nur der Gedanke einer streng kausalen Verknüpfung von Elementarteilchen, den wir aufzugeben haben. Vielmehr ist es der Begriff des körperlichen Teilchens selbst, der in Frage gestellt worden ist; und es erscheint heutzutage unmöglich, in demselben Sinne von kleinsten Teilen der Materie $\mathrm{zu}$ reden, in dem man von Demokrit bis B oltz$\mathrm{m}$ a $\mathrm{n}$ von Atomen gesprochen hat ${ }^{1}$.

Die Schwierigkeiten, auf die ich hier Bezug nehme, sind ja bekannt. Sie entspringen aus der Dualität von Wellen und Korpuskeln, die in de Broglies Entdeckungen zuerst erkannt und später von B o hr als Prinzip der Komplementarität formuliert wurde. Seitdem es gelungen ist, mit einem Elektronenstrahl Interferenzbilder von derselben Art herzustellen, wie sie einmal v. La ue benutzt hat, um die Wellennatur

1 Für eine ausführliche Begründung der folgenden Gedanken sei auf mein Buch Philosophische Grundlagen der Quantenmechanik verwiesen (Deutsche Übersetzung, Verlag Birkhäuser, Basel 1949), das hier als PhGr zitiert wird. Eine allgemeinere Darstellung der philosophischen Ideen, die diesem Buche zugrunde liegen, findet sich in meinem Buch The Rise of Scientific Philosophy (University of California Press, Berkeley und Los Angeles 1951). der Röntgenstrahlen zu beweisen, ist diese Dualität eine experimentelle Tatsache; und wenn wir ein Weltbild der heutigen Physik entwerfen wollen, müssen wir dieser Tatsache Rechnung tragen.

Man hat gesagt, daß es sich hier ja nur um Bilder handelt, die wir uns von den quantenmechanischen Erscheinungen machen; daß Bilder immer unvollkommen sein müssen; und daß es genug ist, wenn solche Bilder dem Physiker bei seinen Arbeiten zu Hilfe kommen, obwohl er sie nicht allzu ernst nehmen sollte. Ich glaube nicht, daß die philosophische Frage, die hier vorliegt, durch derart ausweichende Antworten umgangen werden kann. Wenn sich der Physiker den elektrischen Strom in einem Draht wie einen Wasserstrahl vorstellt und die elektrische Spannung mit dem Druckunterschied des Wassers an verschiedenen Stellen des Rohres vergleicht, so ist das ein Bild. Aber dieses Bild enthebt ihn nicht der Frage, was denn nun der elektrische Strom eigentlich ist. Wenn sich der Physiker die Atome im Kristall wie große farbige Kugeln vorstellt, die auf Drähten aufgespießt sind, so ist das ein Bild; aber dieses Bild erlaubt ihm nicht, der Frage auszuweichen, ob die wirklichen Atome ebenfalls räumlich lokalisierte Volumina sind, wenn auch von kleinerer Dimension und sicherlich ohne Farbe. Solche Fragen sind durchaus vernünftig. Und ich glaube auch, daß sie sich beantworten lassen; nur muß man bereit sein, die Fragen zunächst logisch zu analysieren und Methoden zu entwickeln, welche der Frage eine präzisere Form geben.

Es wäre kurzsichtig, wenn der Physiker solche Fragen einfach ablehnen wollte. Schließlich will er ja nicht nur Formeln besitzen, sondern auch wissen, was sie bedeuten; und er will nicht nur experimentelle Voraussagen machen, sondern auch wissen, welches die allgemeinen Eigenschaften der Natur sind, die sich in diesen Beobachtungsdaten ausdrücken. Der Wunsch des Physikers, von der Beobachtung auf die allgemeinen Eigenschaften der physikalischen Realität zu schließen, ist durchaus berechtigt. Allerdings hat uns die Analyse der Quantenmechanik gelehrt, daß dieser Schluß eine kompliziertere logische Struktur hat, als man früher geglaubt hatte. Die begrifflichen Schwierigkeiten der Quantenphysik rühren daher, daß man die allzu einfache Form der Beziehung zwischen Beobachtung und physikalischer Welt, die für den Makrokosmos besteht, auf den Mikrokosmos zu übertragen versucht hat. Ihre Auflösung kann daher nur erfolgen, wenn man bereit ist, philosophische Schlußweisen zu revidieren. 
Im täglichen Leben bereits begegnen wir dem Unterschied zwischen Dingen, die wir beobachten, und Dingen, die wir erschließen. Dieser Schluß ist meistens sehr einfach: wir sagen, daß die Dinge dieselben sind, ob wir sie beobachten oder nicht. Die letztere Behauptung läßt sich natürlich in keiner Weise beweisen. Wenn man nicht hinschaut, kann man nun einmal die Dinge nicht sehen; und darum kann man auch nicht behaupten, man hätte experimentelle Beweise dafür, daß die unbeobachteten Dinge den beobachteten gleichen. Ich will damit nicht sagen, daß es besser wäre anzunehmen, daß die Dinge jedesmal verschwinden, wenn wir wegblicken. Ich will damit nur sagen, daß die Aussage über die Existenz unbeobachteter Objekte gewisse logische Voraussetzungen enthält, die selbst nicht Beobachtungsresultate sein können.

Solche Sachen hat ja auch $\mathrm{K}$ a $\mathrm{n}$ t schon gesagt; aber ich will nicht in den Fehler verfallen, allgemeine Prinzipien der Realität aus reiner Vernunft deduzieren zu wollen. Wir brauchen keine Metaphysik, wenn wir von physikalischen Objekten sprechen wollen; d. h. wir brauchen keine Prinzipien als wahr anzunehmen, wenn wir über die Beobachtungen hinausgehen wollen. Solche Prinzipien haben vielmehr den Charakter von Definitionen. Wir sagen einfach, daß die Dinge unverändert weiterbestehen, wenn wir nicht hinblicken. Wir haben damit für unsere Sprache eine Erweiterungsregel eingeführt, die uns erlaubt, von beobachteten Dingen zu unbeobachteten überzugehen.

Aber darf man denn das? Natürlich muß man bei solchen Definitionen vorsichtig sein. Wenn mir ein Taschendieb meine Brieftasche stiehlt, während sie sich unbeobachtet in meiner Tasche befindet, kann ich sie nicht wieder hineindefinieren. Die genauere Definition oder Erweiterungsregel lautet: ich will annehmen, daß die physikalischen Gesetze für beobachtete und unbeobachtete Dinge die gleichen sind. Dann kann ich schließen, daß mein Haus auch in der Zwischenzeit an seinem Platz steht, wenn ich es morgens gesehen habe und abends wieder vorfinde; und ich kann gleichfalls schließen, daß meine Brieftasche gestohlen worden ist, wenn ich sie erst in meine Tasche gesteckt habe und nachher nicht mehr vorfinde. Manchmal kann man sogar auf diese Weise schließen, daß die Beobachtung stört, wie z. B. wenn man ein Thermometer in ein Wasserbad steckt; dann kann man ausrechnen, welche Temperatur das Wasserbad vor der Beobachtung hatte.

Das scheint alles sehr trivial zu sein; und doch ist es außerordentlich wichtig. Diese Überlegungen zei- gen uns, daß, wenn wir über eine an sich existierende physikalische Welt sprechen wollen, die von unserer Beobachtung unabhängig besteht, wir zunächst eine bestimmte Sprachform wählen müssen. Diese Sprachform ist keineswegs durch die Wirklichkeit vorgeschrieben. Wenn es jemand vorzieht, zu sagen, daß die Dinge jedesmal verschwinden, wenn er nicht hinschaut, so hat er sich damit für eine andere Sprachform entschieden; d. h. er hat unsere obige Erweiterungsregel abgelehnt und durch eine andere ersetzt. Er darf nur nicht glauben, daß seine Welt inhaltlich von der unseren verschieden ist. Es gibt für die physikalische Welt eine Klasse von gleichwertigen Beschreibungen, die alle gleich wahr sind und sich nur durch die zugrunde liegenden Erweiterungsregeln unterscheiden. Die übliche realistische Sprachform ist nur die einfachste; d. h. sie ist einfacher in dem gleichen Sinn, wie das metrische System einfacher ist als das auf Zoll und Fuß aufgebaute System. Man kann sie das Normalsystem nennen. Daß heißt nicht, daß es keine Wahrheit gibt; es heißt nur, daß man wahre Aussagen auf kompliziertere Weise ausdrücken muß. Ein Sprachsystem zusammen mit seiner Erweiterungsregel ist wahr oder falsch; aber wenn man nicht sagt, welche Erweiterungsregel man benutzt hat, ist das System unvollständig, und man kann überhaupt nicht sagen, ob es wahr oder falsch ist.

Aber auch hier wieder müssen wir eine Vorsichtsmaßregel hinzufügen. Es läßt sich gar nicht a priori voraussagen, ob wir mit unserer Erweiterungsregel durchkommen können. In der klassischen Physik haben wir es stets als selbstverständlich angenommen, daß wir die physikalischen Gesetze von beobachteten Dingen auf unbeobachtete übertragen können. Aber ob sich diese Spracherweiterung widerspruchsfrei durchführen läßt, ist eine empirische Frage. Hier kommen wir an den Punkt, wo sich die Quantenphysik von der klassischen unterscheidet.

Man sagt gewöhnlich, daß wir in der Quantenphysik immer nur Koinzidenzen beobachten. Das ist auch richtig; nur fragt es sich, was für Dinge es eigentlich sind, die da koinzidieren. Ich will deshalb lieber den neutralen Namen Phänomene benutzen. Wenn man sagt, daß es Teilchen sind, die koinzidieren, geht man bereits über den Rahmen der Phänomene hinaus; man sagt damit nämlich, daß das, was wir streng lokalisiert beobachten, auch vorher streng lokalisiert war. Das heißt, man sagt damit etwas darüber aus, wie das Ding aussieht, wenn es nicht beobachtet wird. Solche unbeobachteten Dinge will ich Interphänomene nennen. 
Es wäre irrtümlich, zu glauben, daß wir über Interphänomene nichts aussagen können. Wir können das sehr wohl; nur müssen wir Erweiterungsregeln zu unserer Sprache hinzufügen. Eine sehr zweckmäßige Erweiterungsregel ist z. B. die folgende: Der Wert der beobachteten Größe hat schon vor der Messung bestanden. Diese Regel läßt sich widerspruchsfrei durchführen, und es läßt sich zeigen, daß man damit die Korpuskelinterpretation eingeführt hat ${ }^{2}$. Freilich läßt sich dann ebenfalls zeigen, daß die Messung, die zwar die beobachtete Größe nicht gestört hat, dann eine jede kanonisch konjugierte Größe gestört hat. Die nach $\mathrm{Heisenberg}$ unvermeidliche Störung durch die Beobachtung ist damit sozusagen auf die konjugierte Größe abgeschoben worden. Aber es ist klar, daß damit der Korpuskelinterpretation ein scharf definierter Sinn zugeordnet wird, und daß man deshalb diese Interpretation nicht ein Bild nennen sollte. Sie ist so gut definiert wie jede Wirklichkeitsbeschreibung in der makroskopischen Physik, z. B. wie die realistische Sprache, in der wir sagen, daß Häuser auch dann existieren, wenn man sie nicht sieht.

Die Welleninterpretation läßt sich gleichfalls scharf definieren. Sie kommt darauf hinaus, daß man sagt, die unbeobachtete Größe besitzt gleichzeitig alle Eigenwerte, deren sie überhaupt fähig ist ${ }^{3}$. In einer solchen Definition liegt nichts Paradoxes.

Aber wenn man nun weitergeht und die so eingeführte Korpuskelinterpretation vollständig durchführen will, kommt man zu eigenartigen Schwierigkeiten. Man wird nämlich gezwungen, anzunehmen, daß es eine kausale Wirkung in die Ferne gibt, die sich ohne Zwischenfeld ausbreitet und den entfernten Ort in der Zeit Null erreicht. Ich meine hier die oft diskutierten Überlegungen, nach denen ein Teilchen an einem Schlitz sich verschieden verhält, je nachdem ein anderer Schlitz offen ist, usw. Ich habe diese Beziehungen als kausale Anomalien bezeichnet. Und man kann weiter zeigen, daß für die Welleninterpretation ebenfalls kausale Anomalien auftreten, wenn auch an ganz anderen Stellen. Wenn man geeignete Anordnungen mit Glasplatten benutzt, an denen ein Strahl reflektiert wird, während der andere hindurchgeht, kann man bekanntlich auf diese Weise schließen, daß eine Welle, die gerade am Mond angekommen ist, vernichtet wird, sowie man in einem irdischen Laboratorium eine passende Messung macht.

Man ist geneigt, solche Überlegungen als Unsinn

2 PhGr, S. 132.

3 PhGr, S. 144

$4 \mathrm{PhGr}, \S 26$. abzulehnen. Aber das heißt das Kind mit dem Bad ausschütten. Denn es steckt eine tiefe physikalische Einsicht in solchen logischen Untersuchungen. Es steckt darin das Resultat, daß, wie man auch die Erweiterungsregeln der Sprache konstruiert, man keine Wirklichkeitsbeschreibung erhält, in der sich das Prinzip der Kausalität in normaler Form durchführen läßt; mit anderen Worten, daß es in der Klasse gleichwertiger Beschreibungen der Quantenphysik kein Normalsystem gibt. Dieses Resultat habe ich als Prinzip der Anomalie bezeichnet und an anderer Stelle ganz allgemein aus quantenmechanischen Voraussetzungen bewiesen ${ }^{4}$. Hier liegt der Grund, warum jede erschöpfende Wirklichkeitsbeschreibung der Quantenphysik zu unwillkommenen Konsequenzen führt, und warum der Physiker es oft vorzieht, sich zu weigern, über Interphänomene etwas auszusagen.

Gegen eine solche Weigerung ist auch gar nichts einzuwenden; nur soll man sich über den Grund klar sein. Der Grund ist nicht, daß Aussagen über Interphänomene nicht verifizierbar sind. Auch Aussagen über unbeobachtete Häuser sind unverifizierbar. Sondern der Grund ist, daß, wenn man quantenmechanische Interphänomene nach denselben Prinzipien konstruiert wie unbeobachtete Häuser, man in dem einen Fall zu kausalen Anomalien kommt und in dem anderen nicht. Wenn man in einem Experiment mit zwei Schlitzen für einen Elektronenstrahl ein Interferenzmuster erhalten würde, welches identisch wäre mit der genauen Überlagerung der Muster, die man für jeden Schlitz einzeln erhält, dann würde kein Physiker zögern, Elektronen als ganz gewöhnliche Körperteilchen anzusehen, die sich wie Tennisbälle durch den Raum fortpflanzen - auch wenn es nicht möglich sein sollte, das einzelne Elektron an dem Schlitz ohne Störung zu beobachten. Nur weil wir wissen, daß die Interferenzexperimente ganz andere Resultate zeigen, weigern wir uns, über diese Tennisbälle etwas auszusagen, solange wir sie nicht sehen.

In diesem Zusammenhang möchte ich noch eine weitere Gruppe von kausalen Anomalien erwähnen, deren Bedeutung in einigen neueren Arbeiten hervorgetreten ist. Nach Untersuchungen von $\mathrm{Stückel-}$ berg und Feynman ${ }^{5}$ läßt sich ein Positron als ein Elektron auffassen, das in der Zeit zurückläuft. Hier wird also eine Deutung der Interphänomene durchgeführt, bei der sogar die Zeitrichtung umgekehrt wird; d. h. in der Klasse der gleichwertigen

5 E. C. G. St ückelberg, Helv. physica Acta 14, 588 [1941]; 15, 23 [1942]; R. P. F e y n m a n, Physic. Rev. 76, 749 [1949]. 
Beschreibungen ist die Zeitrichtung nicht mehr eine Invariante. Beobachtungsdaten können uns über die Zulässigkeit einer solchen Deutung nichts lehren; sie sind mit der einen wie mit der anderen Interpretation vereinbar. Und wie in allen derartigen Fällen hat jede der zulässigen Interpretationen neben ihren Nachteilen auch ihre besonderen Vorteile. Wenn man bereit ist, die Umkehr der Zeitrichtung anzunehmen, erkauft man damit den Vorteil, daß das plötzliche Auftauchen und Verschwinden von Elektron-PositronPaaren eliminiert wird: es ist nur ein Elektron da, welches einen Teil seiner Weltlinie in negativer Zeit durchläuft und uns dadurch die Existenz eines Positrons vortäuscht. Hier wird also die Genidentität, d. h. die Identität eines Individuums entlang einer Weltlinie, als eine nichtinvariante Eigenschaft angesehen und damit als die Angelegenheit einer Definition, die in verschiedenen gleichwertigen Beschreibungen in verschiedener Weise gegeben wird. Gegen eine solche Deutung ist logisch nichts einzuwenden; im Gegenteil, sie stellt eine außerordentliche Bereicherung unseres quantenmechanischen Wissens dar und zeigt uns, daß das Prinzip der Anomalie sogar gewisse Umkehrungen des Ursache-Wirkungs-Zusammenhangs einschließt.

Mit diesen Überlegungen kommen wir zu dem Ergebnis, daß in der Quantenphysik eine eigenartige Verschiebung des Wirklichkeitsgehalts physikalischer Theorien vorliegt. Wenn wir die Wirklichkeit vollständig beschreiben wollen, genügt es nicht, eine einzige Beschreibung zu konstruieren. Erst mit der ganzen Klasse gleichwertiger Beschreibungen wird die Darstellung der physikalischen Wirklichkeit vollständig. Solange es ein Normalsystem gibt, wie in der klassischen Physik, genügt es, die Beschreibung in der Sprache des Normalsystems zu geben. Aber wenn es kein solches System gibt, enthält die Aussage, daß es keines gibt, selbst einen wesentlichen Zug der physikalischen Realität; und unsere Darstellung wäre unvollständig, wollten wir diesen Sachverhalt unterdrücken. Wir sind daher gezwungen, neben der physikalischen Sprache noch eine Metasprache zu benutzen, in der wir über die Klasse gleichwertiger Sprachen sprechen; und erst auf diese Weise können wir indirekt eine gewisse Eigentümlichkeit der physikalischen Welt ausdrücken.

Damit wird nun der Grund offensichtlich, warum wir. in der Quantenphysik in Schwierigkeiten kommen, wenn wir über unbeobachtete Objekte sprechen wollen. Jede Aussage über unbeobachtete Objekte enthält implizit eine Aussage über kausale Beziehun- gen; und für quantenmechanische Objekte sind diese kausalen Beziehungen nicht von der einfachen Form, wie sie für klassische Objekte besteht. Wenn wir sagen, daß Häuser auch dann existieren, wenn man sie nicht sieht, dann haben wir damit etwas über die Kausalität ausgesagt; und wenn wir sagen, daß man sich Elektronen nicht als verkleinerte Tennisbälie vorstellen darf, so meinen wir damit, daß solche Teilchen sich nicht in den Rahmen einer Kausalität einordnen lassen, die das Nahwirkungsprinzip befriedigt. Dies ist die Antwort auf die obige Frage, ob die Materie aus räumlich lokalisierten Teilchen besteht: eine solche Aussage hat für sich allein gar keinen Sinn, sondern nur im Zusammenhang mit Aussagen über die Kausalstruktur; und da wir wissen, daß für quantenmechanische Erscheinungen die Kausalität andere Struktureigenschaften besitzt als für makroskopische Erscheinungen, können wir die Gedanken des klassischen Atomismus nicht auf die Quantenphysik übertragen.

Hier wird nun auch der Grund deutlich, warum es sich empfiehlt, in der Quantenmechanik an Stelle der üblichen zweiwertigen Logik eine dreiwertige Logik zu benutzen. Solche logischen Systeme, in denen das tertium non datur aufgegeben wird, sind schon seit ungefähr 30 Jahren bekannt ${ }^{6}$. Aber sie sind zunächst nur als logische Möglichkeiten konstruiert worden, die wie so viele andere mathematische Konstruktionen auf den Augenblick zu warten hatten, wo sich eine physikalische Anwendung für sie fand. Es scheint, daß in der Quantenmechanik zum erstenmal der Fall aufgetreten ist, daß die Physik von einer dreiwertigen Logik Gebrauch machen kann.

In der dreiwertigen Logik gibt es eine Kategorie unbestimmt, die zwischen Wahrheit und Falschheit liegt; und diese Kategorie läßt sich für die quantenmechanischen Interphänomene verwenden. Man sagt dann, daß der Wert der Größe vor der Messung unbestimmt ist; und damit läßt man es zugleich unbestimmt, ob es überhaupt einen einzigen definierten Wert gibt, welcher der ungemessenen Größe zugeschrieben werden kann, wenn man ihn auch nicht kennt. Die Frage, ob die Interphänomene aus Teilchen oder aus Wellen bestehen, ist damit gleichfalls als unbestimmt hingestellt. Durch diese Auffassung gelingt es, die kausalen Anomalien aus dem Gebiet der aussagbaren Sätze auszuschließen.

Selbstverständlich kann eine dreiwertige Logik nur dann als zweckmäßig für die Quantenphysik angesehen werden, wenn man sie auf die Interphäno-

6 Die Literatur ist in PhGr, S. 161-162, angegeben. 
mene bezieht. Für die beobachteten Größen, oder Phänomene, braucht man sie nicht, denn über diese lassen sich ja wahr-falsche Aussagen machen, d. h. Aussagen, die als wahr oder falsch entscheidbar sind. Dies ist von mehreren Physikern mißverstanden worden. Zum Beispiel schreibt B o hr: „Der Gebrauch einer dreiwertigen Logik . . . ist nicht geeignet, die Situation klarer zu beschreiben, da alle wohldefinierten experimentellen Tatsachen ... . in gewöhnlicher Sprache ausgedrückt werden müssen, welche die übliche Logik benutzt“ ${ }^{7}$. Und B or n schreibt: „Die mathematische Theorie, die völlig in der Lage ist, die tatsächlichen Beobachtungen zu erfassen, macht nur von der gewöhnlichen zweiwertigen Logik Gebrauch" ${ }^{8}$. Auch $\mathrm{Pauli}^{9}$ hat ähnliche Äußerungen gemacht. Diese Bemerkungen über die logische Natur der beobachteten Tatsachen sind zweifellos richtig; aber sie treffen das Problem nicht. In der von mir vorgeschlagenen dreiwertigen Logik wird ja gerade die Beobachtungssprache zweiwertig belassen; die dreiwertige Logik wird nur für Interphänomene benutzt ${ }^{10}$. Hier aber erscheint sie unabweisbar. Denn sowie man über das unmittelbar Beobachtete hinausgehen will, treten sonst kausale Anomalien auf.

Es wird manchmal behauptet, daß man es ja nicht nötig habe, die Interphänomene in die Theorie einzubeziehen. Aber die Vertreter einer solchen Auffassung bleiben die Antwort darauf schuldig, warum man in der klassischen Physik die unbeobachteten Größen in die Theorie einbeziehen kann, während dies für die Quantenmechanik zu Schwierigkeiten führt. Mit andern Worten, die auf beobachtete Größen beschränkte Sprache ist nicht reich genug, um alles zu sagen, was man über die physikalische Welt weiß. Ich halte es darum nicht für richtig, zu sagen, daß man die Interphänomene aus der Theorie ausschließen kann. Zum Beispiel ist B ohrs Komplementaritätsprinzip eine Aussage über unbeobachtete Größen, wenn man es in der Form ausspricht: man kann die beobachteten Erscheinungen als Produkt von Wellenoder Korpuskelvorgängen ansehen, und es gibt kein Experiment, welches zwischen diesen beiden Auffassungen entscheiden kann. Diese Aussage wird durch $\mathrm{H}$ e is e $\mathrm{n} b$ ergs Unbestimmtheitsprinzip gestützt; aber sie geht doch insofern über dieses Prinzip hinaus, das sich ja zunächst nur auf Məßresultate be-

7 N. B o hr, Dialectica 2, 317 [1948]. Die Stelle ist von mir aus dem Englischen übersetzt worden.

8 M. B o r n, Natural Philosophy of Cause and Chance, Oxford 1949, S. 107. Diese Stelle ist ebenfalls von mir ïbersetzt worden.

9 W. Pauli, Dialectica 2, 310 [1948]. zieht, als sie behauptet, daß es keinen Sinn hat, der ungemessenen Größe einen bestimmten Wert zuzuschreiben. Damit hat man aber eine metasprachliche Aussage gemacht, in deren Begründung man stillschweigend das Prinzip der Anomalie benutzt hat.

Um dies zu verstehen, denke man an die Eins te in sche Relativitätstheorie, in der man eine bestimmte Gleichzeitigkeitsdefinition benutzt, obwohl man nicht beweisen kann, daß diese Definition in irgendeinem Sinne die „wahre“ Gleichzeitigkeit herstellt. Man kann das tun, weil diese Gleichzeitigkeitsdefinition zu vernünftigen Resultaten führt, nämlich zu einer Zeitordnung, in der das Kausalitätsprinzip nicht verletzt wird. Und es läßt sich sogar zeigen, daß das gleiche für eine gewisse Klasse von Gleichzeitigkeitsdefinitionen gilt, so daß die Auswahl einer bestimmten Definition eine willkürliche Konvention darstellt. In der Quantenmechanik weigert man sich dagegen, irgendeine bestimmte Definition für die Werte unbeobachteter Größen einzuführen, weil jede solche Definition zu kausalen Anomalien führt. Diese Behauptung ist selbst ein wichtiges physikalisches Resultat und sollte deshalb ihren Platz in der Objektsprache der Physik haben. Wenn man die dreiwertige Logik benutzt, kann man auch eine derartige Aussage in der Objektsprache formulieren; sie nimmt dann die Form einer logischen Formel an, die sich in den Zeichen der mathematischen Logik ausdrücken läßt und etwa besagt: Wenn eine quantenmechanische Größe einen gewissen Wert hat, oder nicht hat, dann ist der Wert einer kanonisch konjugierten Größe unbestimmt ${ }^{11}$. Wenn man aber die dreiwertige Logik ablehnt, wird man gezwungen, die Formulierung dieses Gedankens in die Metasprache zu verschieben, wodurch die Theorie eine logisch außerordentlich verwickelte Form erhält.

Um ein anderes Beispiel zu haben, denke man an eine Anordnung, in der ein Elektronenstrahl von sehr geringer Intensität auf eine Blende mit zwei parallelen Schlitzen fällt und ein Interferenzmuster auf einem dahintergestellten Schirm hervorbringt. Wir würden dann gern sagen: ein einzelnes Elektron, das den Schirm erreicht, ist entweder durch den einen oder durch den andern Schlitz gegangen. Wenn man dies

10 Dies gilt allerdings nicht für andere Versuche, die Quantenmechanik in einer mehrwertigen Logik darzustellen. Zum Beispiel bezieht sich die von G. B i rkh off und J. v. N e u m a n n vorgeschlagene mehrwertige Logik der Quantenmechanik (Annals Math. 37, 823 [1936]) -auf Beobachtungsaussagen, und es ist deshalb schwer einzusehen, was mit einer solchen Logik für die Quantenmechanik gewonnen ist.

11 PhGr, S. 173 
als eine Aussage der zweiwertigen Logik auffaßt, kommt man in kausale Anomalien hinein. Wenn man es aber als eine Aussage der dreiwertigen Logik interpretiert, sind die Anomalien ausgeschaltet ${ }^{12}$. B o h r und $\mathrm{H}$ e i s e $\mathrm{n}$ b e r g würden hier nur die Aussage zulassen: Wenn man an jedem Schlitz einen Beobachter aufgestellt hätte, dann hätte nur einer von beiden etwas beobachtet; freilich wäre dann auf dem Schirm nichts passiert. Es ist schwer einzusehen, warum es nun verboten sein soll, etwas Ähnliches für den Fall auszusagen, daß kein Beobachter an den Schlitzen aufgestellt ist und erst auf dem Schirm eine Beobachtung gemacht wird. Wenn man die dreiwertige Logik benutzt, ist eine derartige Aussage statthaft.

Obwohl die Beobachtungssprache zweiwertig ist, steht sie in einem logischen Zusammenhang mit der allgemeineren dreiwertigen Sprache, die auch die Interphänomene umschließt. In jeder Logik gibt es Aussagen von beschränktem Wahrheitsbereich, die sich aus Elementaraussagen eines allgemeineren Wahrheitsbereiches aufbauen lassen. Zum Beispiel kann man in der zweiwertigen Logik durch geeignete Kombination von Elementaraussagen einwertige Aussagen konstruieren, wie die Tautologien, die nur wahr sein können. Ebenso kann man in der dreiwertigen Logik Ausagen konstruieren, die nur wahr oder falsch sein können und darum zweiwertig sind. Ich habe an anderer Stelle ${ }^{13}$ gezeigt, daß gerade diejenigen Aussagen, die wir physikalische Gesetze nennen, in diese Kategorie fallen. Die zweiwertige Sprache der Beobachtungsvoraussage und der Mathematik solcher Aussagen erscheint hier also als eine natürliche Konsequenz in einem allgemeineren logischen System, welches auch unbeobachtete Größen umschließt.

Endlich noch eine Bemerkung über die sogenannte Unentbehrlichkeit der zweiwertigen Logik. Wenn man die Theorie der dreiwertigen Logik durchführt, benutzt man dazu eine Metasprache, die selbst zweiwertig ist. Darin liegt aber kein Zirkelschluß. Vielmehr ist dies ein erlaubtes Verfahren, welches zweckmäßig erscheint, weil wir an den Gebrauch der zweiwertigen Logik nun einmal gewöhnt sind. Dieses Verfahren ist auch gänzlich harmlos, weil man zeigen kann, daß alle logischen Systeme ineinander transformierbar sind. Und es ist ganz gewiß nicht richtig, deshalb die dreiwertige Logik als ein „Spiel mit Symbolen" zu bezeichnen. Der Gebrauch dieser Symbole bringt für den, der sich damit länger be-

12 PhGr, S. 177-178.

13 PhGr, S. 174.

14 Damit ist eine Frage beantwortet, die Born erwähnt hat ${ }^{8}$, S. 108. schäftigt, genau soviel Anschaulichkeit und direktes Verstehen mit sich, wie dies von der nicht-euklidischen Geometrie gilt, die ja auch mit der Welt unserer täglichen Umgebung nicht vereinbar ist ${ }^{14}$.

Dieser Gedanke führt uns in ein weiteres Problem. Man hat die Frage aufgeworfen, ob die Logik einer Sprache eine Angelegenheit willkürlicher Konvention ist, oder ob sie irgendwie die Struktur der Welt zum Ausdruck bringt, auf die sich die Sprache bezieht. Diese Frage läßt sich nicht mit ,ja“ oder „nein“ beantworten, ehe man ihr eine präzisere Form gegeben hat. $\mathrm{Da}$ alle logischen Systeme ineinander transformierbar sind, kann man jede Welt mit jeder Logik beschreiben, und insofern drückt also die Logik keinen Zug der physikalischen Welt aus. Wenn man aber noch weitere Postulate hinzunimmt, wird es anders. Zum Beispiel wenn man verlangt, daß für die Interphänomene keine kausalen Anomalien aussagbar sein sollen, wird man gezwungen, in der Quantenmechanik eine dreiwertige Logik zu benutzen, während das gleiche Postulat für die makroskopische Physik eine zweiwertige Logik erlaubt. Dieses Resultat zeigt, daß die Logik einer Sprache unter gewissen Umständen die Struktur der physikalischen Wirklichkeit widerspiegeln kann; sie wird das immer dann tun, wenn man sie mit gewissen anderen Prinzipien verbindet und von der Sprache verlangt, daß sie dieses System von Prinzipien befriedigt. In diesem Sinne kann man sagen, daß die dreiwertige Logik der quantenmechanischen Sprache diejenige Form verleiht, welche sie zu einem adäquaten Ausdruck der physikalischen Wirklichkeit macht.

Ich möchte diese kurze Darstellung der quantenmechanischen Problemlage mit einer persönlichen Bemerkung beschließen. Seit vielen Jahren ist dies das erstemal, daß ich meine Gedanken zur Philosophie der Physik wieder in einer deutschen Zeitschrift darlege. Es ist mir eine besondere Freude, dies im Rahmen einer Festschrift für meinen verehrten Freund Erich Regener zu tun. Herr Regener war einer der ersten, die meinen Arbeiten zur Philosophie der Physik ein warmes Interesse entgegengebracht haben; und ich bin ihm zu großem Dank verpflichtet für die mannigfache Förderung meiner Arbeiten zu einer Zeit, als die wissenschaftliche Philosophie noch um ihre Existenzberechtigung zu kämpfen hatte. In Bewunderung seiner Leistungen für die Physik und in Dankbarkeit für sein Eintreten für eine Philosophie, die in enger Zusammenarbeit mit der Physik fortschreitet, möchte ich deshalb Herrn Regener meine herzlichsten Wünsche zu seinem 70. Geburtstag aussprechen. 